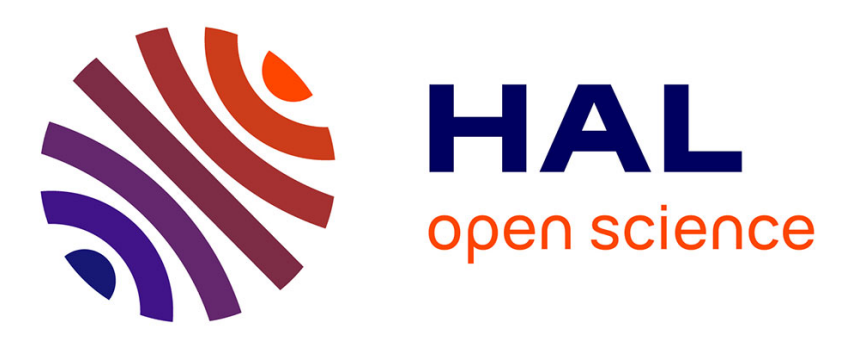

\title{
Influence of speed-related auditory feedback on braking in a 3D-driving simulator
}

\author{
Lionel Bringoux, Jocelyn Monnoyer, Patricia Besson, Christophe Bourdin, \\ Sebastien Denjean, Erick Dousset, Cédric Goulon, Richard \\ Kronland-Martinet, Pierre Mallet, Tanguy Marqueste, et al.
}

\section{To cite this version:}

Lionel Bringoux, Jocelyn Monnoyer, Patricia Besson, Christophe Bourdin, Sebastien Denjean, et al.. Influence of speed-related auditory feedback on braking in a 3D-driving simulator. Transportation Research Part F: Traffic Psychology and Behaviour, 2017, 44, pp.76-89. 10.1016/j.trf.2016.10.006 . hal-01436173

\section{HAL Id: hal-01436173 \\ https://hal.science/hal-01436173}

Submitted on 31 Oct 2019

HAL is a multi-disciplinary open access archive for the deposit and dissemination of scientific research documents, whether they are published or not. The documents may come from teaching and research institutions in France or abroad, or from public or private research centers.
L'archive ouverte pluridisciplinaire HAL, est destinée au dépôt et à la diffusion de documents scientifiques de niveau recherche, publiés ou non, émanant des établissements d'enseignement et de recherche français ou étrangers, des laboratoires publics ou privés. 


\title{
Influence of speed-related auditory feedback on braking in a 3D-driving simulator
}

\author{
Anca Stratulat ${ }^{\mathrm{b}}$ \\ ${ }^{a}$ Aix-Marseille Université, CNRS, ISM, UMR 7287, France \\ ${ }^{\mathrm{b}}$ PSA Peugeot-Citroen, Velizy-Villacoublay, France \\ ' Safran Engineering Services, Saclay, France \\ ${ }^{\mathrm{d}}$ Aix-Marseille Université, Centrale Marseille, CNRS, LMA, UPR 7051, France
}

Lionel Bringoux $^{\mathrm{a}, *}$, Jocelyn Monnoyer ${ }^{\mathrm{a}, \mathrm{b}}$, Patricia Besson ${ }^{\mathrm{a}, \mathrm{c}}$, Christophe Bourdin ${ }^{\mathrm{a}}$, Sébastien Denjean $^{\mathrm{b}}$, Erick Dousset ${ }^{\mathrm{a}}$, Cédric Goulon ${ }^{\mathrm{a}}$, Richard Kronland-Martinet ${ }^{\mathrm{d}}$, Pierre Mallet $^{\mathrm{a}}$, Tanguy Marqueste $^{\mathrm{a}}$, Cécile Martha ${ }^{\mathrm{a}}$, Vincent Roussarie ${ }^{\mathrm{b}}$, Jean-François Sciabica ${ }^{\mathrm{b}}$,

\section{A R T I C L E I N F O}

\section{Article history:}

Received 11 January 2016

Received in revised form 8 September 2016

Accepted 4 October 2016

\section{Keywords:}

Driving

Speed regulation

Sensory feedback

Auditory

Sound synthesis

Simulation

\begin{abstract}
A B S T R A C T
Although discrete auditory stimuli have been found useful for emergency braking, the role of continuous speed-related auditory feedback has not been investigated yet. This point may though be of importance in electric vehicles in which acoustic cues are drastically changed. The present study addressed this question through two experiments. In experiment 1,12 usual drivers were exposed to naturalistic auditory feedback mimicking those issued from electric cars, while facing dynamic visual scenes in a 3D driving simulator. After being passively travelled up to a sustained constant speed, subjects had to stop their car in front of a traffic light that unexpectedly turned to red. Modifications of the speedrelated auditory feedback did not impact braking initiation and regulation. In experiment 2 , synthesized auditory feedback based on the Shepard-Risset glissando was provided to a new sample of 15 usual drivers in the same task. Pitch variations of this acoustic stimulus, although not scaled to an absolute speed, were manipulated as a function of visual speed changes. Changing the mapping between pitch variations of the synthesized auditory feedback and visual speed changes induced adjustments on braking which depended on acceleration/deceleration feedback. These findings stressed the importance of the acoustic content and its dynamics for car speed control.
\end{abstract}

(c) 2016 Elsevier Ltd. All rights reserved.

\section{Introduction}

With the commercialization of electric vehicles at a larger scale, there is a growing interest regarding the impact of sound on driving. Indeed, electric vehicles produce little sound, due to the lack of internal combustion engine noise. This improves comfort for drivers and their environment. However, it also results in the drastic reduction of source of information regarding car displacement. While this can obviously impact the safety of pedestrians and other road users such as cyclists, it also raises the question of how this reduced auditory feedback could directly affect driving. Since driving safety has mostly been associated with drivers perception and control of their actual speed (Aarts \& van Schagen, 2006; Brooks et al., 2011; Horswill

\footnotetext{
* Corresponding author at: Institut des Sciences du Mouvement (EJ Marey), 163 Avenue de Luminy - Case 901,13009 Marseille, France.

E-mail address: lionel.bringoux@univ-amu.fr (L. Bringoux).
} 
\& McKenna, 1999; Joksch, 1993), it appears crucial to investigate how acoustic content during driving may influence drivers speed perception and its effective regulation.

Actually, both presence and level of internal noise have been found critical for visual speed judgments. In the absence of acoustic feedback, or when this feedback is reduced, drivers tend to underestimate their speed to a greater extent (Evans, 1970; Horswill \& Plooy, 2008; Matthews \& Cousins, 1980; Merat \& Jamson, 2011). For instance, based on psychometric estimates, Horswill and Plooy (2008) showed that a $5 \mathrm{~dB}$ attenuation of the ambient sound led to a decrease in perceived visual speed of about $5 \mathrm{~km} \cdot \mathrm{h}^{-1}$ (for a $60 \mathrm{~km} \cdot \mathrm{h}^{-1}$ reference visual speed). However, a critical question remains to be solved before the auditory influence on road safety can be assessed: While the level of ambient sound may affect subjective speed estimates, does this necessarily impact driving behavior? Although recent findings supported a direct link between motion perception scores and driving performance, including braking responses (Wilkins, Gray, Gaska, \& Winterbottom, 2013), the specific consequences of sound manipulation on driving control (and not only on speed estimates) has not yet been investigated.

Currently, auditory-based Advanced Driving Assistance Systems (ADAS) provided in modern vehicles are mostly focused on discrete alerting stimuli which may help drivers to trigger earlier braking reactions (Chang, Lin, Fung, Hwang, \& Doong, 2008; Mckeown, Isherwood, \& Conway, 2010). This appears all the more effective when the intensity of such alerting stimuli is directly referred to the time to collision as for looming auditory warnings (Gray, 2011).

The question arises as to whether continuous speed-related auditory feedback could provide more subtle and smoother speed regulation than discrete or collision-related signals provided to the drivers. In other words, whether different types of online auditory feedback directly referred to car speed changes may impact braking is a relevant issue which has remained unexplored to our knowledge.

The present study therefore aims at investigating the direct influence of continuous auditory feedback on visual speed regulation through a braking task presented in a 3D-driving simulator. Specifically, the question was addressed via two experiments, in which the content of the acoustic information was manipulated, either using naturalistic sound recordings resembling those which would be emitted by an electric vehicle, or using synthesized sounds evoking motion dynamics through pitch variations. Overall, we hypothesized that sound content and its inherent dynamics conveyed by acoustic variations could be decisive for influencing driving in terms of speed regulation and braking.

\section{General methods}

The main device was composed of a fixed-based driving simulator inserted into a 3D immersive environment (Fig. 1). The virtual reality display (CAVE for Cave Automatic Virtual Environment) corresponded to a $3 \mathrm{~m}$ wide, $4 \mathrm{~m}$ high box space with three vertical screens constituting walls and a horizontal screen for the floor. The three vertical surfaces were back-projected, and the ground received direct projection with a $1400 \times 1050$ pixel resolution and a $60 \mathrm{~Hz}$ frame rate (more details about this setup can be found in Bringoux et al., 2009). In-house software (Ice $\odot$ ) was used to build and control virtual scenarios. It enabled projection of a virtual scene composed of a dynamic driver's perspective observed from a car travelling along a straight two-lane road. The driving simulator included a brake pedal with a mechanical gauge to control online the deceleration of the virtual car, from which visual and auditory cues were displayed and adjusted in real-time. Drivers' actions and simulator responses were linked directly without transformation (e.g.., no simulated inertia, no skid, straight handling). Maximal deceleration capabilities were standardized in both experiments at $16 \mathrm{~m} \cdot \mathrm{s}^{-2}$.

In both following experiments, the virtual car was first passively accelerated up to a $90 \mathrm{~km} \cdot \mathrm{h}^{-1}$ (i.e., $25 \mathrm{~m} \cdot \mathrm{s}^{-1}$ ) constant visual speed (Fig. 2). For all trials and conditions, the level of the initial passive acceleration was set at $3.25 \mathrm{~m} \cdot \mathrm{s}^{-2}$. In the

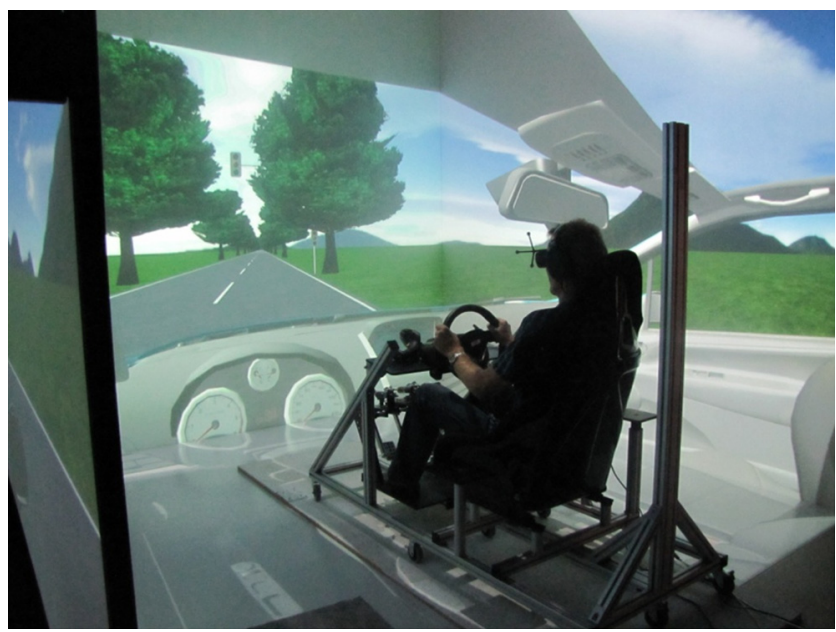

Fig. 1. Illustration of the main setup. A driving simulator was placed in a CAVE-like environment to provide realistic 3D visual stimuli to which different auditory stimuli were scaled and displayed to the drivers via earphones. 
constant speed section, green traffic lights were positioned along the road at $40 \mathrm{~m}$ intervals. When the car was either $80 \mathrm{~m}$ or $40 \mathrm{~m}$ from a light, the light could turn red. This was done either after a pseudorandom driving duration at constant speed (Panels A and B; Fig. 2) or at the end of the acceleration phase, i.e., as soon as the car reached $25 \mathrm{~m} \cdot \mathrm{s}^{-1}$ (Panels C and D; Fig. 2). The first scenario was used in both experiments, whereas the second was used in Experiment 2 only. The driver
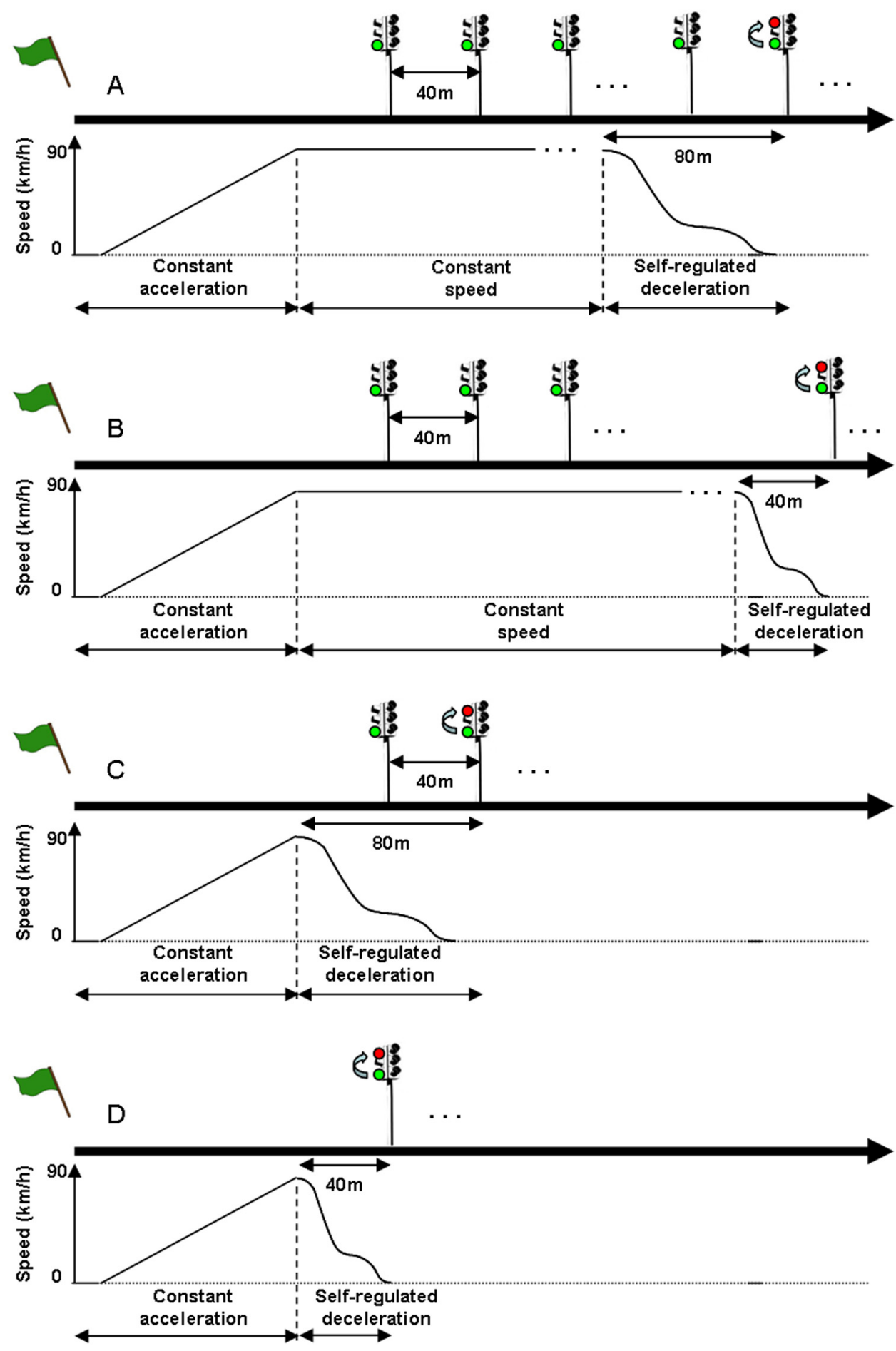

Fig. 2. Schematic representation of the procedure used for both experiments. Upper panels [A and B]: sequence of events leading to braking after travelling at constant speed, with an $80 \mathrm{~m}$ (Panel A) or $40 \mathrm{~m}$ (Panel B) stopping distance. Panels A and B correspond to situations presented in Experiments 1 and 2 . Lower panels [C and D]: sequence of events leading to braking at the end of the passive acceleration ramp, with an $80 \mathrm{~m}$ (Panel C) or $40 \mathrm{~m}$ (Panel D) stopping distance. Panels C and D correspond to situations presented in Experiment 2 only. 
was instructed to brake as soon as the light turned red and bring the car to a stop at the light (i.e., with the front bumper aligned with the post of the traffic light).

Car position over time was recorded at a sample acquisition frequency of $60 \mathrm{~Hz}$. The car position signal was low-pass filtered (Butterworth filter; frequency cut-off: 2; order: 2) and derivatives taken to get speed and acceleration signals over time (Fig. 3). Car final position (CFP) was defined as the signed distance separating the front of the stationary vehicle after braking from the center of the post of the light. Braking initiation/termination were defined as the time when vehicle speed dropped below $24.99 \mathrm{~m} \cdot \mathrm{s}^{-1}$ and $0.01 \mathrm{~m} \cdot \mathrm{s}^{-1}$ respectively, following the constant speed section, enabling reaction time (RT) and braking time (BT) to be calculated. Peak deceleration (PD) as well as the normalized time-to-peak deceleration relative to braking duration (rTPD) were extracted from the acceleration signal.

For each experiment, all the experimental conditions were presented in a pseudo-random order. Statistical analyses were performed using repeated-measures analyses of variance (ANOVAs), after having carefully ensured that normality and variance homogeneity of the data were respected, hence allowing parametric comparisons. Post-hoc tests (Newman-Keuls) were also performed when necessary. The level of significance was set at .05 for all statistical analyses.

\section{Experiment 1}

\subsection{Participants}

Twelve young healthy subjects participated in this experiment ( 7 males, 5 females; mean age: 25.9 \pm 6.4 ). They had all held a driving licence for at least 2 years and were regular drivers (minimum twice a week). They declared no history of previous sensory or motor disorder. They had normal audition and normal vision or corrected-to-normal vision. All of them signed a written informed consent form prior to the study, in accordance with the 1964 Declaration of Helsinki and the study had the written consent of a local institutional review board (IRB) which specifically approved it.

\subsection{Experimental conditions}

Two optic flow conditions were used (poor vs. rich optic flow). Specifically, low vs. high informational content relative to optical edge rate (i.e., the rate at which local visual discontinuities cross a fixed point of reference in the observer's field of view) and optical texture (Fajen, 2005) was provided via the absence or presence of roadside trees and whether the line in the middle of the road was solid or broken. A traffic light was set to switch from green to red only once the car had been travelling at $25 \mathrm{~m} \cdot \mathrm{s}^{-1}$ for at least $8 \mathrm{~s}$. This was done to provide a sufficient standardized experience of constant speed to the drivers. In addition, the light turned red when the car was either $40 \mathrm{~m}$ or $80 \mathrm{~m}$ away from the light, hence defining two stopping distances (i.e., emergency vs. controlled).

A realistic ambient sound reproducing aerodynamic and rolling noises (thus simulating the sound produced by an electric vehicle) was displayed to the driver via headphones (HD 650 Sennheiser( ). For this, several acoustic stimuli were recorded binaurally at various speeds in an internal combustion engine vehicle (Peugeot 308®) with the engine turned off, using an artificial head recording system (HSM III Head Acoustics@). Sound segments were interpolated using Audacity® software

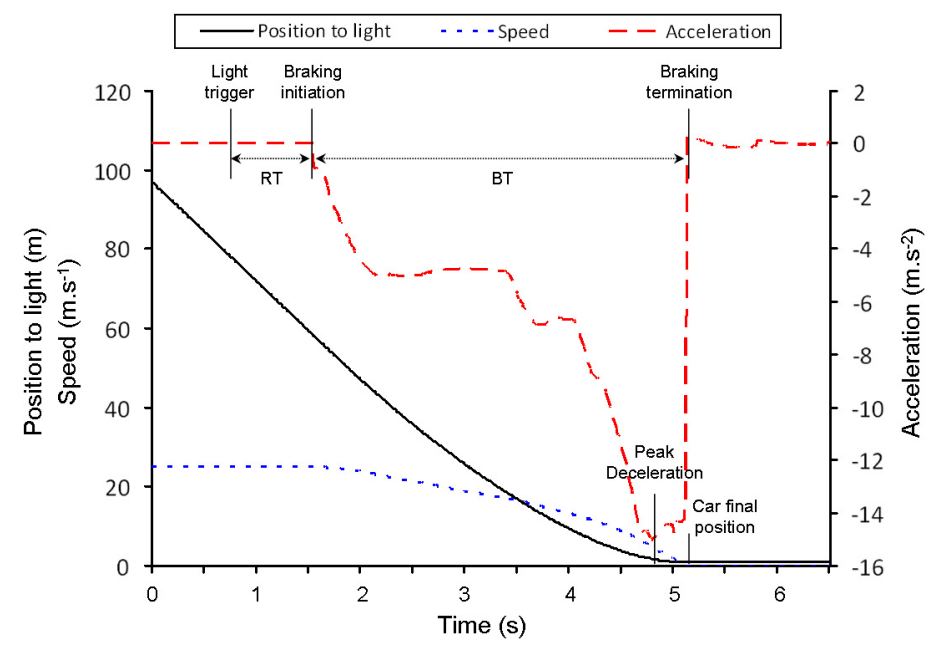

Fig. 3. Typical plots representing car position to light (black bold line), speed (blue dotted line), and acceleration (red dashed line) as a function of time, for a single trial recorded in Experiment 2 (Trigger event: constant speed; Stopping distance: 80 m; SSSF: Mapping 2; see methods). Raw parameters allowing for subsequent kinematics processing are also indicated (Light trigger; Braking initiation; Peak Deceleration; Braking termination). (For interpretation of the references to colour in this figure legend, the reader is referred to the web version of this article.) 
and scaled relative to the 3D visual stimuli using in-house virtual reality software (Ice $\subset$ ). The displayed sound corresponded either to the simulated visual speed of the driving simulator or to a higher or lower speed (up to 20\% discrepancy). In other words, the drivers were provided sounds that corresponded to equal, faster or slower speeds than they actually drove. Different naturalistic Speed-related Sound Scale Factors (SSSF; i.e., "audiovisual gains") were thus manipulated. For instance, a SSSF of 1.1 means here that the sound displayed for a given visual speed actually corresponded to a sound recorded at a $10 \%$ higher speed. Coincident sound level variations were measured using a digital sound level meter (SL-4022 REED@) held next to the microphone. The extreme auditory ratios ranged from 65.9 to $70.1 \mathrm{~dB}$ when the visual speed was set at $25 \mathrm{~m} \cdot \mathrm{s}^{-1}$. In summary, for this experiment, ANOVAs were performed on the selected variables (see general methods) for 2 visual scenes (poor vs. rich optic flow) $\times 2$ stopping distances $(40$ vs. $80 \mathrm{~m}) \times 5$ naturalistic speed-related sound scale factors (SSSF: 0.8 ; $0.9 ; 1 ; 1.1 ; 1.2$ of visual speed).

\subsection{Results}

\subsubsection{Car Final Position (CFP)}

With regard to the participants ability to stop the car at the traffic light, the ANOVA showed a main effect of stopping distance, $F(1,11)=4.71, p<.05$. The shorter the stopping distance, the more forward the CFP (closer to the light, as drivers stopped on average before the target, see Fig. 4). There were no other main effects and no interactions between all factors regarding this variable, $p>.05$. The analysis of within-subject standard deviation (SD) with regard to car final position did not reveal any significant main effects or interactions between any factors.

\subsubsection{Reaction Time (RT) and Braking Time (BT)}

Regarding the time to trigger and the time to execute braking, the ANOVA showed a main effect of stopping distance for RT, $F(1,11)=24.76, p<.001$, and BT, $F(1,11)=48.05, p<.001$, but no other main effects and no interactions between any factors appeared for these two variables. The shorter stopping distance led to a shorter reaction time and braking time (Fig. 5). RT and BT within-subject SD were lower for the shorter stopping distance, $F(1,11)=28.47, p<.001$ and $F(1,11)=24.12$, $p<.001$ for RT and BT respectively, but no other main effects or interactions between any factors regarding RT and BT variability were significant.

\subsubsection{Peak Deceleration (PD) and relative Time-to-Peak Deceleration (rTPD)}

Analysis of car kinematics during braking revealed that the stopping distance also significantly impacted PD, $F(1,11)=49.15, p<.001$. The shorter stopping distance led to a higher peak deceleration (Fig. 6A). Noticeably again, no other main effects or interactions between any factors were significant regarding PD. The ANOVA conducted on within-subject SD also revealed a significant decrease in PD variability with the shorter stopping distance, $F(1,11)=9.36, p<.01$, but as for previous observations, no other main effects or interactions between any factors regarding PD variability were significant. When focusing on the normalized time-to-peak deceleration relative to braking time (rTPD), the analysis did not reveal any significant main effects or interactions between any factors (Fig. 6B). In other words, the temporal organisation of braking was unaffected by the various experimental conditions.

\subsection{Intermediate discussion (Experiment 1)}

The key purpose of this first experiment was to investigate whether speed-related modifications of naturalistic aerodynamic and rolling noises could change the braking behavior of drivers. Overall, among all the experimental conditions used,

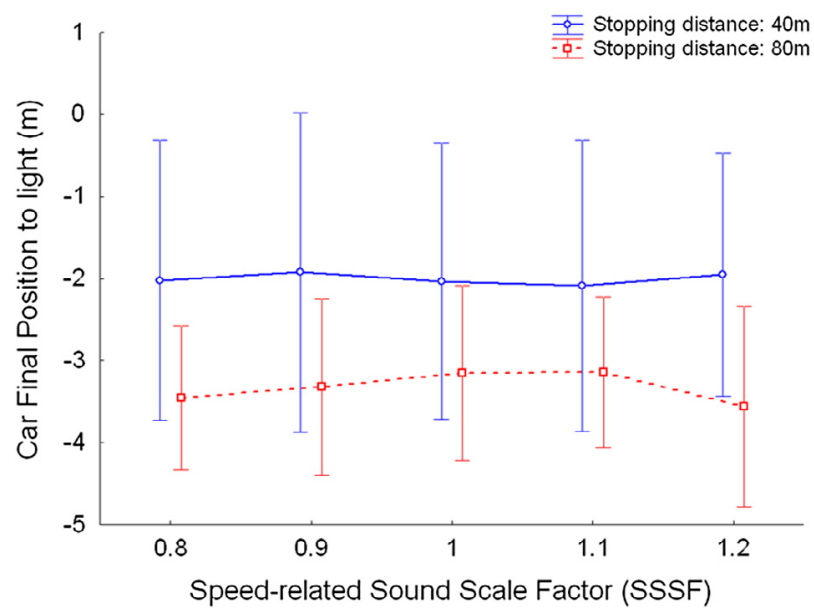

Fig. 4. Car final position to light (CFP) as a function of realistic SSSF for both stopping distances. Error bars represent the 95\% confidence interval. 

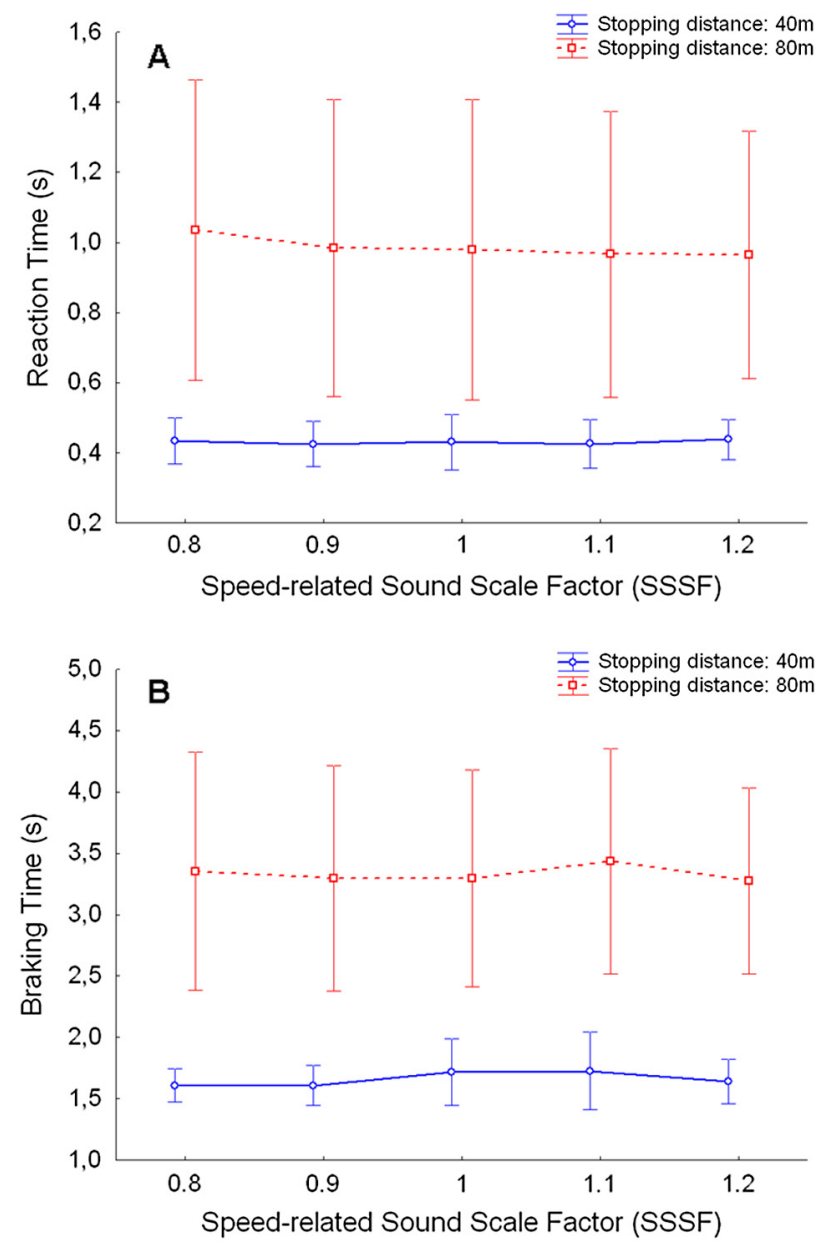

Fig. 5. Reaction time to braking (RT; Panel A) and braking time (BT; Panel B) as a function of realistic SSSF for both stopping distances. Error bars represent the $95 \%$ confidence interval.

stopping distance appears to be the only independent variable likely to impact braking. Indeed, the shorter stopping distance ( $40 \mathrm{~m}=$ emergency braking) led the driver to stereotypically react earlier, and brake stronger within a decreased time interval, nevertheless leading to a more forward car final position (i.e., closer to the traffic light) compared with the longer stopping distance. This is in line with observations reported when drivers are requested to respect a sufficient braking distance to avoid collisions (Konstantopoulos, Chapman, \& Crundall, 2010).

On the other hand, use of a poorer visual scene in the present experiment had no consequence on braking preparation and performance. This is somewhat in contradiction with some reports which clearly showed a strong impact of visibility (Hills, 1980; Muhrer \& Vollrath, 2010), and optic flow (DeLucia \& Tharanathan, 2009; Fajen, 2005; Fajen, 2008) on driving. However, a recent work unambiguously demonstrated that drivers did not slow down significantly until visibility distance was drastically reduced (Brooks et al., 2011), suggesting that the visual deterioration used here was insufficient to modify braking behavior.

In addition - and most importantly with regard to the present objectives, use of a naturalistic sound comparable to that produced by an electric car was here unable to modify braking when strictly referred to visual scene kinematics. In other words, a $\pm 20 \%$ change in speed-related aerodynamic/rolling noises displayed to the driver is insufficient to convey informational content susceptible to deteriorate or improve driver performance. The amount of change of the acoustic stimulus level is, however, of the same order as in Horswill and Plooy's study (Horswill \& Plooy, 2008) on perceived speed (from 67.5 to $72.5 \mathrm{~dB})$.

Thus, it makes good sense to ask whether the absence of acoustic influence on speed regulation was due to its intrinsic content rather than its change in terms of sound level. Although auditory perception of car speed was initially found to rely on sound level (Gatehouse \& Frankie, 1980), several recent findings have suggested that sound "structure" could participate in defining speed perception. For instance, it has been shown that the frequency content of engine noise can modulate 

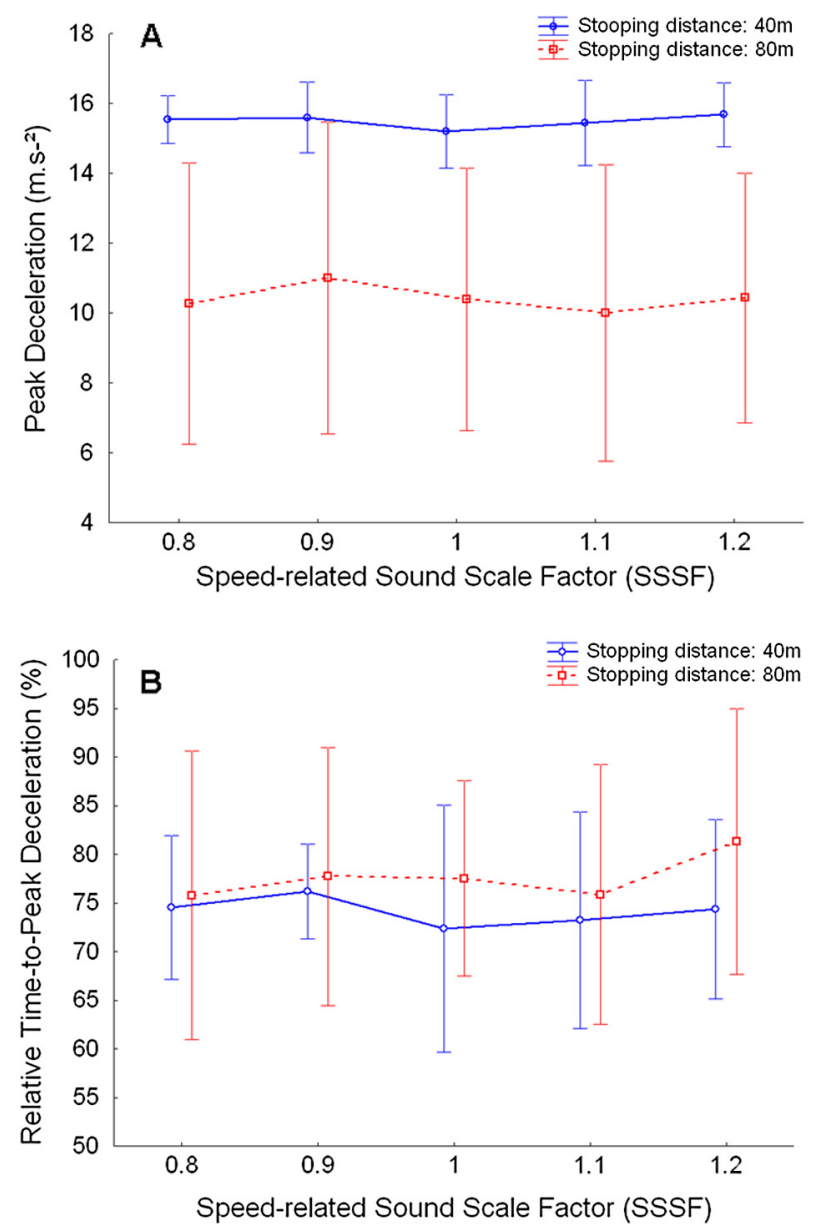

Fig. 6. Peak deceleration (PD; Panel A) and relative time-to-peak deceleration (rTPD in \% of the braking duration; Panel B) as a function of realistic SSSF for both stopping distances. Error bars represent the 95\% confidence interval.

drivers perception of speed changes (Roussarie, Richard, \& Bezat, 2004; Sciabica, 2011). This issue related to sound content is specifically tackled in the following experiment.

\section{Experiment 2}

\subsection{Participants}

Fifteen young healthy subjects participated in Experiment 2 ( 7 males, 8 females; mean age: $26.9 \pm 2.2$ ). None of them took part in Experiment 1. The same prerequisites for participants inclusion used in Experiment 1 were adopted for this second experiment.

\subsection{Experimental conditions}

Compared with Experiment 1, only one visual scene was presented (i.e., poor optic flow condition). The traffic light was set to switch from green to red either as soon as the car reached the end of the acceleration ramp or when it had been traveling at constant speed for at least $8 \mathrm{~s}$, thus defining two light trigger events. This event also occurred when the car was either $40 \mathrm{~m}$ or $80 \mathrm{~m}$ away from the changing light, thus defining two stopping distances as in the previous experiment. Each trial was performed either without any sound or accompanied by aerodynamic and rolling noises simulating an electric vehicle plus a synthesized sound developed to give further information on vehicle dynamics to the driver. Based on the metaphor of internal combustion engine noise, the pitch variations of this sound were correlated to vehicle speed changes. The higher the magnitude of the acceleration or deceleration, the faster the pitch increased or decreased respectively. This sound was generated in a manner similar to the Shepard-Risset infinite glissando (Shepard, 1964), using a Gaussian-filtered harmonic comb, allowing continuous pitch variations to be maintained over long accelerations. The pitch could be controlled via two 
parameters: the central frequency of the Gaussian window, which varied exponentially from $120 \mathrm{~Hz}$ at idle to $600 \mathrm{~Hz}$ at $36 \mathrm{~m} \cdot \mathrm{s}^{-1}$, and the harmonic components of frequency variation at a speed directly mapped to the vehicle speed variations.

Therefore, sound content was here unrelated to a specific visual speed level (i.e., no absolute matching) contrary to the previous experiment. However, the pitch variation of the sound was directly correlated to visual speed changes (i.e., relative matching). With an arbitrary reference mapping (Mapping 1), corresponding to a pitch variation of one octave per $12.5 \mathrm{~m} \cdot \mathrm{s}^{-1}$ of vehicle speed variation, two other speed-related sound scale factors were tested. They were obtained by either dividing by 2 (Mapping 0.5) or multiplying by 2 (Mapping 2) the pitch variation with respect to visual speed changes. In addition, aerodynamic and rolling noises from a combustion engine car (Peugeot 308@) were recorded binaurally with the engine turned off and played back at the corresponding speed using a granular synthesis technique. Acoustic feedback was generated using the Hartis synthesizer developed by PSA Peugeot-Citroen $\odot$ and displayed to the driver via headphones (HD280 Pro Sennheiser $($ ), whose sound level was controlled online with respect to car speed.

Hence, for this experiment, 2 light trigger events (end of acceleration vs. constant speed) $\times 2$ stopping distances (40 vs. $80 \mathrm{~m}$ ) $\times 4$ synthesized speed-related sound scale factors (SSSF: No sound; Mapping 0.5; Mapping 1; Mapping 2) ANOVAs were performed on the selected variables (see general methods).

\subsection{Results}

\subsubsection{Car Final Position (CFP)}

Analyses performed on CFP revealed a main effect of the stopping distance, $F(1,14)=28.86, p<.001$. The shorter stopping distance led to a more forward CFP (closer to the traffic light with drivers also stopping before the target on average, see Fig. 7). The ANOVA also yielded a main effect of SSSF, $F(3,42)=12.58, p<.001$, and, most importantly, a significant interaction between SSSF and stopping distance, $F(3,42)=15.48, p<.001$. As illustrated in Fig. 7 , the presence of sound (regardless of content) led to a more backward CFP for the shorter stopping distance (no sound vs. all auditory mappings: $p<.001$ ). Conversely for the longer stopping distance, the higher mapping between sound and vision led to a more forward CFP as compared with the lower mapping, $p<.001$ and to the no sound condition, $p<.05$. The ANOVA did not reveal any significant main effect of the trigger event or any interactions with the other factors. Within-subject SD regarding CFP also decreased with the shorter stopping distance, $F(1,14)=5.97, p<.05$ and when sound was available, regardless of content, $F(3,42)=5.11$, $p<.01$ (no sound vs. "increasing" auditory mappings of $0.5,1,2: p<.05, p<.01, p<.01$, respectively). There was no main effect of the trigger event or interactions between any factors regarding within subject variability for this parameter.

\subsubsection{Reaction Time (RT) and Braking Time (BT)}

Analysis on RT, which corresponds to braking preparation, revealed a main effect of the trigger event, $F(1,14)=4.78$, $p<.05$, a main effect of the stopping distance, $F(1,14)=86.56, p<.001$ and a main effect of the synthesized SSSF, $F(3,42)=13.46, p<.001$. Notably, significant interactions were found between trigger event and stopping distance, $F$ $(1,14)=30.09, p<.001$ as well as between trigger event and SSSF, $F(3,42)=2.85, p<.05$. The longer stopping distance led to a higher RT. This effect was more pronounced when the stimulus-to-brake was triggered just at the end of the acceleration phase (Fig. 8A).

Interestingly, the presence of sound (regardless of mapping) significantly decreased RT values (no sound vs. all auditory mappings, $p<.001$ ), and this effect was more pronounced when the stimulus-to-brake was triggered just at the end of the acceleration phase (Fig. 8B). RT individual variability was affected by the stopping distance (i.e., within-subject SD was

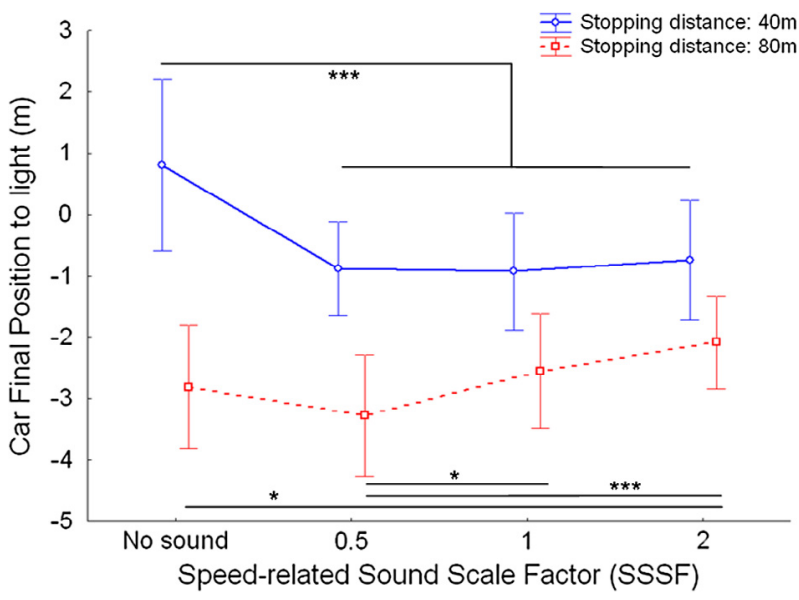

Fig. 7. Car final position to light (CPF) as a function of synthesized SSSF for both stopping distances. Error bars represent the 95\% confidence interval. Stars represent the level of significance for statistical differences $\left({ }^{*} \mathrm{p}<.05 ;{ }^{* * *} \mathrm{p}<.001\right)$. 

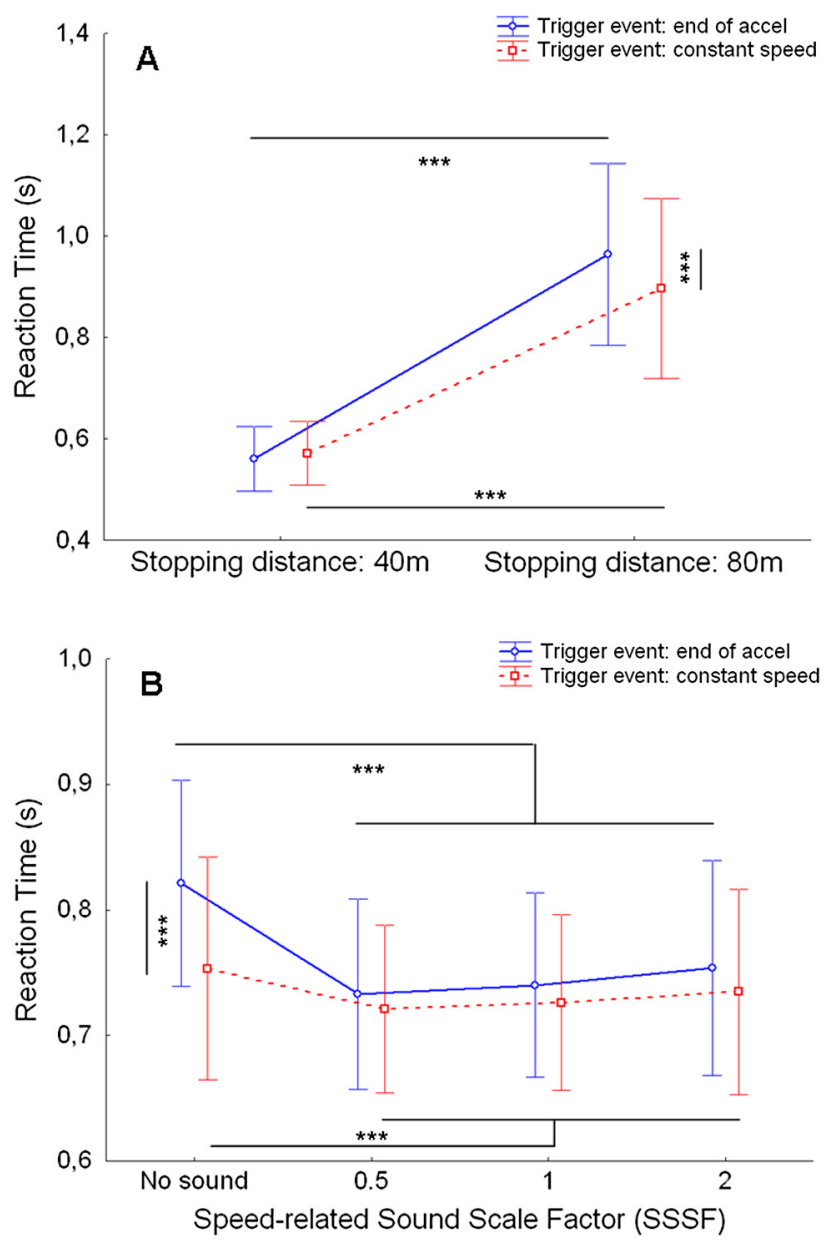

Fig. 8. Reaction time to braking (RT) as a function of stopping distance (Panel A) and synthesized SSSF (Panel B) for both trigger events. Error bars represent the $95 \%$ confidence interval. Stars represent the level of significance for statistical differences $\left({ }^{* * *} \mathrm{p}<.001\right)$.

significantly higher for the longer stopping distance, $F(1,14)=19.75, p<.001$, but no other main effects or interactions between any factors regarding RT variability were significant.

Analysis on BT, which corresponds to braking performance, revealed a main effect of the trigger event, $F(1,14)=7.58$, $p<.05$, a main effect of the stopping distance, $F(1,14)=164.45, p<.001$ and a main effect of SSSF, $F(3,42)=3.43, p<.05$. Notably, significant interactions were found between trigger event and stopping distance, $F(1,14)=7.85, p<.05$ as well as between stopping distance and SSSF, $F(3,42)=6.61, p<.001$. Unsurprisingly, the longer stopping distance led to a higher BT, this effect being more pronounced when the stimulus-to-brake was triggered after constant speed (Fig. 9A). More notably, whereas the presence of sound and variations in auditory mapping did not yield any significant effects on braking time for the shorter stopping distance, $p>.05$, the ANOVA revealed a significant influence of sound for the longer stopping distance (Fig. 9B).

The higher the auditory mapping, the lower the BT, except compared with the no-sound condition. When considering individual variability on BT, a significant interaction was found between stopping distance and SSSF. Indeed, the presence of sound decreased within-subject SD on BT only for the shortest stopping distance at auditory mappings of 0.5 and 2 , $p<.05$. Neither main effects nor interactions between any other factors regarding BT variability were significant.

\subsubsection{Peak Deceleration (PD) and relative Time-to-Peak Deceleration (rTPD)}

Analysis on PD during braking revealed a main effect of the trigger event, $F(1,14)=5.32, p<.05$, a main effect of the stopping distance, $F(1,14)=17.89, p<.001$ and a main effect of SSSF $F(3,42)=9.67, p<.001$. Notably, significant interactions were found between trigger event and stopping distance, $F(1,14)=9.28, p<.01$ as well as between stopping distance and SSSF, $F$ $(3,42)=8.68, p<.001$. The longer stopping distance led to a lower PD, and this effect was more pronounced when the stimulus-to-brake was triggered at constant speed (Fig. 10A).

More strikingly, whereas the presence of sound and variations in auditory mapping did not yield any significant effects on PD for the shorter stopping distance, $p>.05$, the ANOVA revealed a significant influence of sound for the longer stopping 

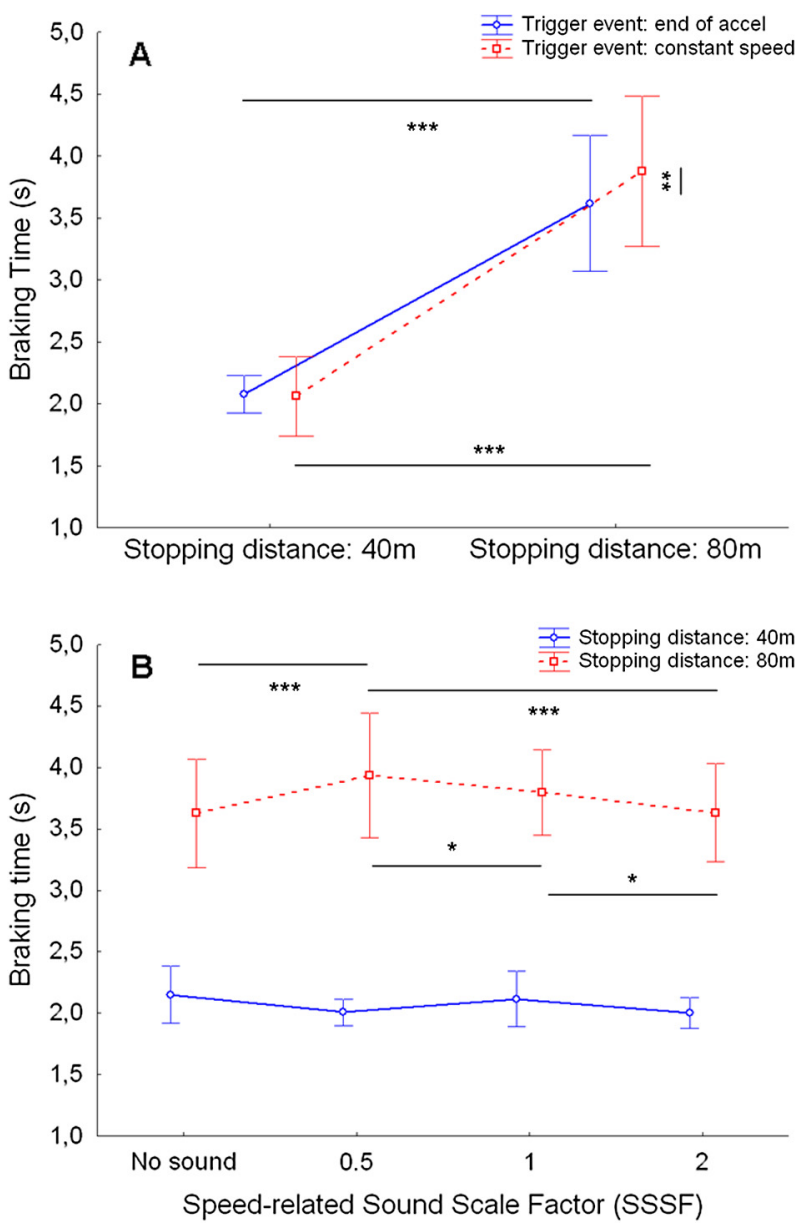

Fig. 9. Panel A: Braking time (BT) as a function of stopping distance for both trigger events. Panel B: BT as a function of synthesized SSSF for both stopping distances. Error bars represent the $95 \%$ confidence interval. Stars represent the level of significance for statistical differences $\left({ }^{*} \mathrm{p}<.05 ;{ }^{* *} \mathrm{p}<.01 ;{ }^{* * *} \mathrm{p}<.001\right)$.

distance (Fig. 10B). Indeed, the lower auditory mapping decreased PD and the higher auditory mapping increased PD compared with the no sound condition. Furthermore, the higher the auditory mapping, the higher the PD. PD individual variability was affected by the stopping distance (i.e., within-subject SD was significantly higher for the longer stopping distance, $F(1,14)=19.75, p<.001)$, but no other main effects or interactions between any factors regarding PD variability were significant.

Analyses conducted on the relative time-to-peak deceleration (rTPD) revealed a main effect of the stopping distance, $F(1,14)=31,7, p<.001$, and, most importantly, a significant interaction between stopping distance and SSSF, $F(3,42)=8.15, p<.001$. As illustrated in Fig. 10C, whereas increasing auditory mapping led to an earlier peak deceleration for the shorter stopping distance, it conversely delayed rTPD for the longer stopping distance.

\subsection{Intermediate discussion (Experiment 2)}

As in Experiment 1, stopping distance influenced braking behaviour, but this effect was mainly modulated by the moment at which the light turned to red. Indeed, a longer stopping distance allowed the driver to brake more smoothly (i.e., lower peak deceleration) within a longer amount of time, especially when the light trigger was set after constant speed (i.e., a comfortable cruise-like condition). A longer distance for braking also allowed the driver to react more slowly. This effect was magnified when the light trigger was set at the end of the acceleration phase. In that situation, the fact that the event occurred at the earliest moment (in the range of possible trigger occurrences) could constitute an element of surprise which may affect reaction time. Overall, car motion kinematics (e.g., speed variations vs. cruise condition) have unsurprisingly been found to be crucial to the way drivers react to unexpected events on the road (Vollrath, Schleicher, \& Gelau, 2011).

The key finding of the second experiment lies in how sound was found to impact speed regulation. All the extracted variables were affected by the use of the synthesized sound. During emergency braking (i.e., for the shorter stopping distance), the presence of sound led to more cautious behaviour, mostly independent of the applied auditory mapping. This is 

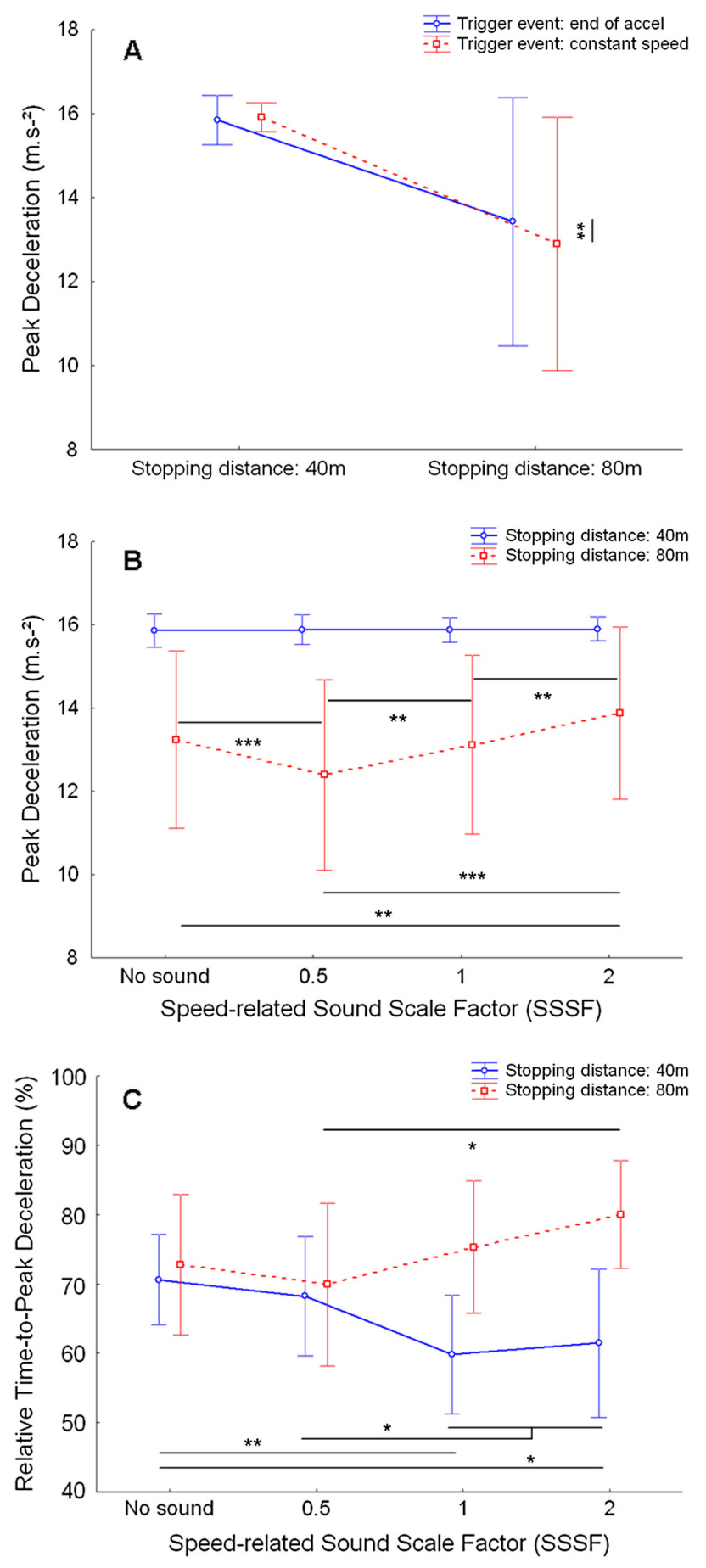

Fig. 10. Panel A: Peak Deceleration (PD) as a function of stopping distance for both trigger events. Panel B: PD as a function of synthesized SSSF for both stopping distances. Panel C: relative time-to-peak deceleration (rTPD in \% of the braking duration) as a function of synthesized SSSF for both stopping distances. Error bars represent the 95\% confidence interval. Stars represent the level of significance for statistical differences $\left({ }^{*} \mathrm{p}<.05 ;{ }^{* *} \mathrm{p}<.01 ;{ }^{* * *} \mathrm{p}<.001\right)$.

supported by a shorter reaction time (similar for all auditory mappings) and a shorter relative time-to-peak deceleration (except for the lower auditory mapping), which enabled the driver to stop the car before the light when synthesized auditory feedback was provided. Conversely, when the stopping distance was longer, providing the driver with enough time to perform the task, more subtle regulations were observed. In this case, while the presence of sound, regardless of its content, also lowered reaction time, increasing the auditory mapping induced a delayed relative time-to-peak deceleration, which was compensated for by a higher peak deceleration, leading to a shortened braking time. In other words, when a comfortable 
distance is allowed for braking, the variation of sound dynamics during car deceleration also interacts with visual speed perception: a larger decrease in sound pitch during initial deceleration (e.g., Mapping 2) may lead the driver to perceive car deceleration as higher than it actually is. As a consequence, he/she may underestimate the actual instantaneous speed and accordingly perform the main braking "later but stronger" on approaching the traffic light. These points, put in perspective with the findings from Experiment 1, will be further discussed in the following section.

\section{Discussion}

The present study was designed to address the influence of speed-related auditory feedback on braking, this feedback comprising either naturalistic sound recordings comparable to those produced by electric cars, or synthesized sounds whose pitch variations are linked to motion dynamics. At first glance, the two experiments yielded contradictory results with regard to acoustic influence on braking behaviour. Firstly, various explanations that may help understanding of why speed-related changes of aerodynamic and rolling noises alone had no impact on braking initiation and regulation will be reviewed. Secondly, the specific influences observed when using synthesized auditory feedback which was also speed-related, but not scaled to specific visual velocities, will be considered. Finally, applied considerations will be addressed regarding the design of ADAS in modern cars, taking into account the main findings of the present study.

\subsection{Realistic aerodynamic and rolling noises variations did not impact braking}

Braking is a critical driving behavior based on speed regulation, which has been found to be highly related to perceived speed (Horswill \& McKenna, 1999). For this reason, as perceived speed has been found to be affected by changes in auditory feedback issued from car recordings (Horswill \& Plooy, 2008), a main and significant influence of acoustic information on overall braking behavior was expected. This was clearly not the case in Experiment 1: the sound variations applied did not modify any parameters of the braking kinematics. Notably in this experiment, the range of sound level variations ( $\sim 5 \mathrm{~dB}$, see Methods) was comparable to the one used in Horswill \& Plooy' study (2008). This observation thus excludes sound level as a key factor to explain the difference in sound influence found in the two studies. Rather, the specific sound content and its interactions with other experimental factors might be advanced as reasons. Firstly, isolated aerodynamic and rolling noises are rarely experienced by drivers of cars fitted with internal combustion engines. It is plausible that prior exposure to these noises could be necessary for such auditory feedback to become informative (Kobayashi, Teramoto, Hidaka, \& Sugita, 2012; McKeown \& Isherwood, 2007). More critically, the frequency variations characterizing aerodynamic and rolling noises across the various sound scale factors were substantially lower than those which would be obtained using sounds from internal combustion vehicles. The question arises as to whether the frequency component of the acoustic information displayed, and specifically its dynamics, constitutes a relevant and meaningful source of information about speed changes (Denjean et al., 2013). At this stage, it cannot be excluded that sound variations like those issued from electric cars could become informative with increasing vehicle speed and gaps between visual and auditory-evoked speeds. Nevertheless, the results from the second experiment gave some hints as to the way acoustic structure and dynamics could play a major role in speed estimates and speed control during braking.

\subsection{Contextual influences of sound contents and dynamics offered by sound synthesis}

Interestingly, displaying synthesized sound including rapid pitch variations as a function of speed changes led to significant braking modulations. These modulations were notably different when comparing emergency braking situations (i.e., shorter stopping distance) and more comfortable conditions during which braking could be regulated (i.e., longer stopping distance). Firstly, the overall influence of sound revealed during emergency braking was characterized by a lower reaction time to braking and an earlier time-to-peak deceleration, leading the driver to stop the car more backward from the light (see intermediate discussion for Experiment 2). It is hypothesized that this cautious behaviour may be derived from an overestimation by drivers of car speed before the braking trigger in the presence of auditory feedback. The results indeed suggest that the level of speed reached at the end of the acceleration ramp was perceived as higher than it actually was when the synthesized sound was present, regardless of its mapping relative to optic flow. Here, it seems important to emphasize sound dynamics for the emergence of such an effect. The synthesized sound variations induced during initial acceleration may be processed by the central nervous system with visual inputs to yield an amplified speed sensation, which remains even after a sustained period of constant speed (as suggested by the absence of interaction between trigger event and speed-related sound scale factor). The overall impact of sound dynamics upon speed estimates has already been suggested in a previous work showing that temporal acoustic irregularities (i.e., sound pulses) may provoke an arousal effect allowing drivers to decrease their reaction time under simulated driving conditions (Suied, Susini, \& McAdams, 2008).

More strikingly, the present results suggest that sound dynamics may also operate during car deceleration, leading to more subtle influences on speed regulation when drivers have enough time to brake (i.e., longer stopping distance). Indeed, increasing the rate of pitch variation of the synthesized sound may also lead to an enhanced deceleration sensation. In other words, the drivers might initially consider braking too hardly when they start to regulate their speed, this effect being stronger with higher auditory mapping (i.e., with faster initial sound pitch decrease). Consequently, they might proportionally 
delay the occurrence of the main braking phase (as attested by a longer relative time-to-peak deceleration), but compensate for this delay by braking more strongly (i.e., higher peak deceleration) when becoming aware of proximity to the traffic light. This strategy led however the drivers to stop the car somewhat further forward. In this situation, sound dynamics mainly exerted an influence on braking during the deceleration phase when the drivers had time to integrate this information into behavioural regulations.

Several recent findings have shown that movement perception and control may be strongly influenced by continuous variations of a synthesized sound, as has been reported for instance in functional rehabilitation and learning (Danna et al., 2014; Rodger, Young, \& Craig, 2014). Nevertheless, the specific influence of each speed-related sound attribute has yet to be thoroughly tested, including tone, pitch, timbre, and specific perceptual effects such as the ones corresponding to the Risset illusion or Phasing (Kronland-Martinet, Ystad, \& Aramaki, 2012). To our knowledge, however, this is the first time that the manipulation of sound content has been so closely linked to driving behavior. This may have important repercussions in the design of human-machine interfaces in automotive research and the way acoustic information could be implemented in modern vehicles (e.g. hybrid or electric).

\subsection{Application for automotive research and development}

Although some previous applied experiments have revealed a positive influence of sound on braking by using discrete alerting auditory stimuli (Chang et al., 2008; Mckeown et al., 2010), we are not aware of any study which demonstrated specific contextual influences on driving of continuous speed-related auditory feedback produced by sound synthesis. Firstly, providing such synthesized auditory feedback appears highly beneficial to driving safety during emergency braking, as it favours faster reactive responses comparable to those observed for "alerting" stimuli. Secondly, beyond enhancing contextual awareness about driving state (Janssen, Iqbal, \& Ju, 2014; Walker, Stanton, \& Young, 2008), the continuous synthesized sound used here participated in fine speed regulation, especially when a sufficient amount of time was allowed for the drivers to incorporate its variations. Noticeably however, while faster sound pitch variations may yield relative speed overestimation following acceleration, the same faster pitch variations may conversely yield speed underestimation following deceleration. Thus, from a behavioural standpoint, it is highly important to consider "directional" aspects of sound dynamics when providing speed-related auditory feedback in electric cars. ADAS designers could for instance implement reversible mappings as a function of car acceleration/deceleration to take into account the different effects sound manipulation has on braking. Finally, the present data support the idea that sound content has a significant impact on speed regulation. Appropriate synthesized sounds may thus intuitively help controlling hybrid or electric vehicles without learning to interpret aerodynamic/rolling noise. Specifically, dynamic properties of sound could be better suited for immediately informing about speed variations rather than absolute speed itself. Indeed, while the exclusive use of auditory feedback may become relevant for cueing drivers about relative speed changes, we argue that it may become unreliable for reaching and keeping a given speed value (e.g., drive to reach and maintain $90 \mathrm{~km} \cdot \mathrm{h}^{-1}$ ) because of the absence of a natural scaling between sound and speed. Overall, our findings have also to be considered in relation with the subjective preferences of drivers which may also define sound acceptance in modern vehicles (Wogalter, Lim, \& Nyeste, 2014). Furthermore, the experience level and age of drivers could affect their ability to integrate this new informational content, a hypothesis which must be tested in future studies. At last, the validity of our results has to be challenged in more dynamic contexts, where inertial cues (i.e., vestibular/somatosensory inputs) are available to the drivers.

\section{Conclusion}

The key conclusion of the present study could be stated as follows: modulating naturalistic sounds such as those audible in electric cars seems unable to affect braking initiation and control in a 3D-driving simulator. On the other hand, providing synthesized sound displaying unambiguous speed-scaled pitch variations can influence the way drivers initiate and regulate braking, presumably by helping them better access information about vehicle speed changes.

\section{Acknowledgements}

This work was supported by a Grant from ISM-PSA OpenLab Automotive Motion Lab. The authors would like to thank the Mediterranean Virtual Reality Center staff (CRVM) for technical assistance.

\section{References}

Aarts, L., \& van Schagen, I. (2006). Driving speed and the risk of road crashes: A review. Accident Analysis and Prevention, 38(2), 215-224.

Bringoux, L., Bourdin, C., Lepecq, J.-C., Sandor, P. M. B., Pergandi, J.-M., \& Mestre, D. (2009). Interaction between reference frames during subjective vertical estimates in a tilted immersive virtual environment. Perception, 38(7), 1053-1071.

Brooks, J. O., Crisler, M. C., Klein, N., Goodenough, R., Beeco, R. W., Guirl, C., ... Beck, C. (2011). Speed choice and driving performance in simulated foggy conditions. Accident Analysis and Prevention, 43(3), 698-705.

Chang, S.-H., Lin, C.-Y., Fung, C.-P., Hwang, J.-R., \& Doong, J.-L. (2008). Driving performance assessment: Effects of traffic accident location and alarm content. Accident Analysis and Prevention, 40(5), 1637-1643.

Danna, J., Fontaine, M., Paz-Villagrán, V., Gondre, C., Thoret, E., Aramaki, M., ... Velay, J.-L. (2014). The effect of real-time auditory feedback on learning new characters. Human Movement Science, 43, 216-228. 
DeLucia, P. R., \& Tharanathan, A. (2009). Responses to deceleration during car following: Roles of optic flow, warnings, expectations, and interruptions. Journal of Experimental Psychology: Applied, 15(4), 334-350.

Denjean, S., Velay, J. L., Kronland-Martinet, R., Roussarie, V., Sciabica, J.-F., \& Ystad, S. (2013). Are electric and hybrid vehicles too quiet for drivers? In Proceedings of Internoise, Innsbruck, 15-18 September.

Evans, L. (1970). Speed estimation from a moving automobile. Ergonomics, 13(2), 219-230.

Fajen, B. R. (2005). The scaling of information to action in visually guided braking. Journal of Experimental Psychology. Human Perception and Performance, 31 (5), 1107-1123.

Fajen, B. R. (2008). Perceptual learning and the visual control of braking. Perception E Psychophysics, 70(6), 1117-1129.

Gatehouse, R. W., \& Frankie, G. H. (1980). The development of an auditory concept of speed. The Journal of Genetic Psychology, 136(2nd Half), 221-229.

Gray, R. (2011). Looming auditory collision warnings for driving. Human Factors, 53(1), 63-74.

Hills, B. L. (1980). Vision, visibility, and perception in driving. Perception, 9(2), 183-216.

Horswill, M. S., \& McKenna, F. P. (1999). The development, validation, and application of a video-based technique for measuring an everyday risk-taking behavior: Drivers' speed choice. The Journal of Applied Psychology, 84(6), 977-985.

Horswill, M. S., \& Plooy, A. M. (2008). Auditory feedback influences perceived driving speeds. Perception, 37(7), 1037-1043.

Janssen, C. P., Iqbal, S. T., \& Ju, Y.-C. (2014). Sharing a driver's context with a caller via continuous audio cues to increase awareness about driver state. Journal of Experimental Psychology: Applied, 20(3), 270-284.

Joksch, H. C. (1993). Velocity change and fatality risk in a crash - A rule of thumb. Accident Analysis and Prevention, 25(1), $103-104$.

Kobayashi, M., Teramoto, W., Hidaka, S., \& Sugita, Y. (2012). Indiscriminable sounds determine the direction of visual motion. Scientific Reports, 2, 365.

Konstantopoulos, P., Chapman, P., \& Crundall, D. (2010). Driver's visual attention as a function of driving experience and visibility. Using a driving simulator to explore drivers' eye movements in day, night and rain driving. Accident Analysis and Prevention, 42(3), 827-834.

Kronland-Martinet, R., Ystad, S., \& Aramaki, M. (2012). High level control of sound synthesis for sonification processes. AI \& Society, Springer, 27(2), 245-255.

Matthews, M., \& Cousins, L. (1980). The influence of vehicle type on the estimation of velocity while driving. Ergonomics, $23(12), 1151-1160$.

McKeown, D., \& Isherwood, S. (2007). Mapping candidate within-vehicle auditory displays to their referents. Human Factors, 49(3), 417-428.

Mckeown, D., Isherwood, S., \& Conway, G. (2010). Auditory displays as occasion setters. Human Factors, 52(1), 54-62.

Merat, N., \& Jamson, H. (2011). A driving simulator study to examine the role of vehicle acoustics on drivers' speed perception. In Proceedings of the 6th International Driving Symposium on Human Factors in Driver Assessment, Training and Vehicle Design (pp. 226-232). California: Olympic Valley - Lake Tahoe.

Muhrer, E., \& Vollrath, M. (2010). Expectations while car following: the consequences for driving behaviour in a simulated driving task. Accident Analysis and Prevention, 42(6), 2158-2164.

Rodger, M. W. M., Young, W. R., \& Craig, C. M. (2014). Synthesis of walking sounds for alleviating gait disturbances in Parkinson's disease. IEEE Transactions on Neural Systems and Rehabilitation Engineering: A Publication of the IEEE Engineering in Medicine and Biology Society, 22(3), 543-548.

Roussarie, V., Richard, F., \& Bezat, M.-C. (2004). Perceptive qualification of engine sound character, validation of auditory attributes using analysis-synthesis method. In Proceedings CFA/DAGA, Strasbourg, France.

Sciabica, J. F. (2011). Perceptual characterization of interior car noise PhD dissertation. Aix-Marseille University.

Shepard, R. N. (1964). Circularity in judgements of relative pitch. Journal of Acoustic Society of America, 36(12), $2346-2353$.

Suied, C., Susini, P., \& McAdams, S. (2008). Evaluating warning sound urgency with reaction times. Journal of Experimental Psychology: Applied, 14(3), $201-212$.

Vollrath, M., Schleicher, S., \& Gelau, C. (2011). The influence of cruise control and adaptive cruise control on driving behaviour - a driving simulator study. Accident Analysis and Prevention, 43(3), 1134-1139.

Walker, G. N., Stanton, N., \& Young, M. (2008). Feedback and driver situation awareness (SA): A comparison of SA measures and contexts. Transportation Research Part F: Traffic Psychology and Behaviour, 11(4), 282-299.

Wilkins, L., Gray, R., Gaska, J., \& Winterbottom, M. (2013). Motion perception and driving: Predicting performance through testing and shortening braking reaction times through training. Investigative Ophthalmology E' Visual Science, 54(13), 8364-8374.

Wogalter, M. S., Lim, R. W., \& Nyeste, P. G. (2014). On the hazard of quiet vehicles to pedestrians and drivers. Applied Ergonomics, 45(5), 1306-1312. 